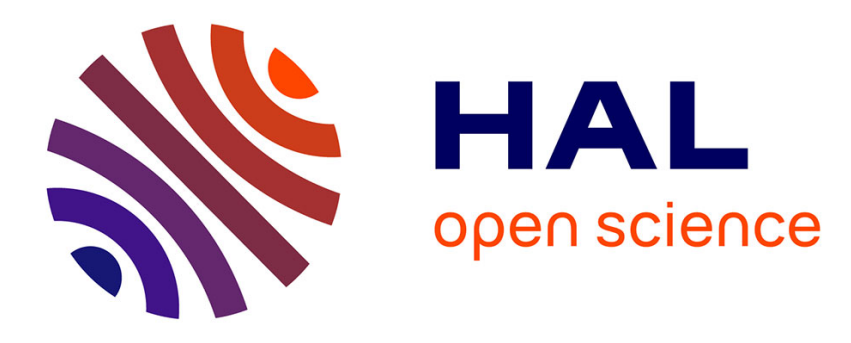

\title{
Acoustics and frictional sliding in granular materials
}

Sean Mcnamara

\section{To cite this version:}

Sean Mcnamara. Acoustics and frictional sliding in granular materials. Granular Matter, 2015, 17 (3), pp.311. 10.1007/s10035-015-0563-3 . hal-01154388

\section{HAL Id: hal-01154388 \\ https://hal.science/hal-01154388}

Submitted on 2 Jun 2015

HAL is a multi-disciplinary open access archive for the deposit and dissemination of scientific research documents, whether they are published or not. The documents may come from teaching and research institutions in France or abroad, or from public or private research centers.
L'archive ouverte pluridisciplinaire HAL, est destinée au dépôt et à la diffusion de documents scientifiques de niveau recherche, publiés ou non, émanant des établissements d'enseignement et de recherche français ou étrangers, des laboratoires publics ou privés. 


\title{
Acoustics and Frictional sliding in Granular materials
}

\author{
Sean C. McNamara
}

Received: April 3, 2015

\begin{abstract}
We study numerically the propagation of an acoustic pulse through a loaded granular material under the hypothesis that the conventional modeling of solid friction used in soft sphere discrete element modeling remains valid at acoustic time scales. As a pulse crosses the material, it temporarily suppresses sliding contacts, making it difficult to prepare states that correspond to experimental conditions. The pulse speed is strongly affected by the loading in a very anisotropic way, varying by as much as a factor of two depending on the propagation direction. We separate the contribution of the contact network from that of sliding contacts, and show that sliding contacts can reduce the propagation speed as much as changes in coordination number. Sliding contacts have a characteristic acoustic signature: pulse speed depends on sign (compression or rarefaction), even at very small amplitudes.
\end{abstract}

\section{Introduction}

Several recent experiments use acoustical waves to probe the deformation granular materials $[1 ; 2 ; 3 ; 4 ; 5]$ : changes in the acoustic transmission properties are shown to coincide with deformation events [2] or changes in imposed shear [1]. These techniques give information about the interior of the packing while remaining non-intrusive, and will probably become more widespread in the future.

The weakness of these methods lies in their interpretation. One observes that acoustical changes are correlated to deformation, but it is not clear exactly how.

S. McNamara

Institut de Physique de Rennes, UMR UR1 - CNRS 6251, Université de Rennes I, Bât 11A, 35042 Rennes Cedex, FRANCE
Explanations usually advanced are changes in the coordination number [1] or, in other situations, contact nonlinearity [2]. Recently, other possible mechanisms have been proposed: intergranular collisions, frictional slip, and excitations of small groups of grains or force chains [6].

One possible way forward is to examine numerical simulations. While simulations are necessarily simplistic, they do allow for precise control of parameters and detailed examination of results. If certain acoustical phenomena could be reproduced in simulations, one could then determine their origin, for in simulations, one has access to all the physical variables. Accordingly, some numerical studies of granular acoustics have appeared $[7 ; 8 ; 9 ; 10 ; 11 ; 12 ; 13]$. These works focus on the propagation of a pulse through a packing subjected to an isotropic stress, and focus on the geometrical disorder of granular materials that prevents the application of methods used in crystals.

But one often wants to probe packings subjected to an anisotropic stress or undergoing deformation, where sliding contacts play an important role. Recent experimental works $[14 ; 15]$ have shown that the interaction between sound waves and tangential forces is complex. However, to our knowledge, this work is the first numerical study of the interaction between sliding and acoustics.

The numerical model of the tangential force used here is very simplistic: it uses only a single, linear, stiffness, and a constant friction ratio. Thus this work cannot describe many effects such as contact ageing, sound wave-induced changes in friction ratio [14], or nonlinear contact stiffness. Instead, this work studies one effect, namely, the interaction between the sliding-nonsliding transition and acoustics. As will be shown, this interaction can be very strong. 
This study must also confront the great difference the acoustic frequencies and the strain rate. Granular material are probed at frequencies of order $100 \mathrm{kHz}$, whereas typical shear rates are of order $10^{-5} \mathrm{~Hz}[1]-\mathrm{a}$ difference of ten orders of magnitude. It is not possible to resolve both time scales in the same way in a numerical model. But we show how this difficulty can be overcome using the elementary soft sphere "molecular dynamics" method.

This paper is organized as follows. In Sec. 2, we explain and motivate the numerical setup, and introduce the different numerical techniques used in the article. In Sec. 3, we then examine the effect of sound waves on sliding contacts, whereas the effect of sliding contacts on acoustic properties is examined in Sec. 4. The paper concludes with Sec. 5 where experimental evidence for the effects found in this paper are discussed.

\section{Numerical setup}

\subsection{Biaxial tests and Checkpoints}

We study two-dimensional quasi-static packings of $N=$ $128 \times 128=16384$ grains confined by four walls, The grains have a two-dimensional mass density $\rho_{*}$, and the total mass of the packing is $M_{*}$. The packing was formed by compressing a granular gas. During the compression, the bottom and left walls were fixed, while the upper and right walls were mobile. A constant pressure $p_{*}$ was applied to them, and their motion was obtained by integrating Newton's second law, as if they were grains with a mass of about $M_{*} / 100$. During compression, the grains were frictionless, and a weak, diagonally directed gravitational force was applied to push rattlers against the granular skeleton.

In this way, a very dense motionless state is obtained, that will serve as the initial condition of the biaxial test. Friction is "turned on" by setting the friction ratio $\mu=0.2$, although grain-wall interactions remain frictionless. Note that all tangential forces vanish, as the packing was constructed without friction. This is different from the experimental initial condition where friction is always active. A constant velocity is imposed on the upper wall, but the right wall remains mobile, with the same pressure $p_{*}$ applied to it. Fig. 1 shows two resulting stress-strain curves that are typical for this kind of numerical experiment. The curve marked "compression" is obtained by moving the upper wall downward, and the curve marked "extension" is obtained by moving the upper wall upward.

As the packing is being deformed, a "checkpoint" is written into a file periodically at regular strain intervals $\Delta \varepsilon=10^{-5}$. A checkpoint is a complete snapshot of

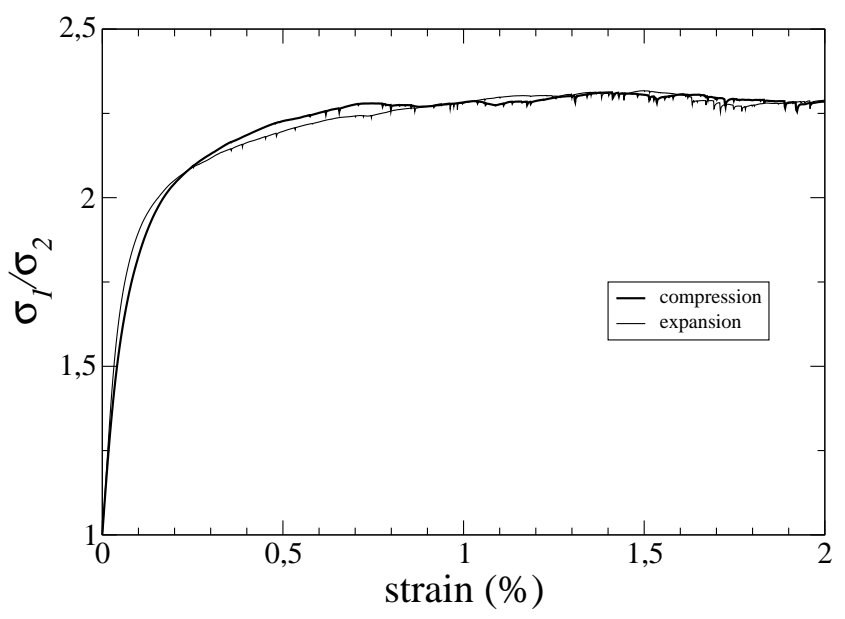

Fig. 1 Stress-strain curve for the simulations studied in this paper. The first principal stress is $\sigma_{1}$ and $\sigma_{2}$ is the second principal stress. In the compression test, the upper wall is lowered, and $\sigma_{1}=\sigma_{y y}>\sigma_{2}=\sigma_{x x}=p_{*}$. In the extension test, the upper wall is raised, and $\sigma_{1}=\sigma_{x x}=p_{*}>\sigma_{2}=\sigma_{y y}$.

the system that can be used to restart the simulation. Their usual purpose is to make the simulation program more robust. If there is a power cut in the middle of a long simulation, one does not need to restart the simulation from the beginning; instead one can restart the simulation from the last checkpoint recorded. In this paper, checkpoints are an essential part of the numerical method, enabling us to probe a state at different amplitudes, and to modify the imposed strain rate.

\subsection{Signal generation}

To acoustically probe the sample, we reload a checkpoint, and restart the simulation. A signal is generated by adding a perturbation on the constant force on the right hand wall:

$p(t)=p_{*}[1+f(t)]$.

The same checkpoint can be reloaded many times, and pulses with different amplitudes can be generated each time. In this way, we can apply different signals to exactly the same packing. If necessary, we can apply a zero-amplitude pulse, i.e., simply replay the simulation without any change at all.

\subsection{Why compression and extension?}

We consider both compression and extension tests because the acoustic properties a granular material subjected to an anisotropic stress are also anisotropic. As will be described above in Sec. 2.2, the mobile wall is 
used to generate signals that propagate only in the horizontal direction, the vertical direction being inaccessible. To overcome this difficulty, we will assume that the vertical direction in the compression test is equivalent to the horizontal direction in the extension test.

To support this assumption, and to illustrate the anisotropy, we generate a wave at the center of the sample, and examine its propagation. This is done by reloading a checkpoint, and then multiplying the radius of one grain near the center by $1+10^{-5}$ before beginning the simulation. This sudden change of radius generates a perturbation in the packing that radiates outward. The velocities of all the particles are recorded at a high sampling rate.

To identify the changes introduced by the perturbation, a second simulation is done with the same initial conditions, except no particle radius is changed. The particle velocities are recorded in exactly the same way as in the first simulation. Then, the perturbed velocity $\mathbf{v}$ of each grain is obtained by subtracting the results of the second simulation from the first.

When we examine the perturbed velocity of each grain, we find that it is zero until a well defined time when it suddenly begins to fluctuate. We interpret the appearance of these fluctuations as the arrival of the disturbance generated at the center. The arrival time is estimated as the time when $|\mathbf{v}|$ first rises to $10 \%$ of its maximum value. Doing this for each grain in three different simulations yields Fig. 2 .

The level of gray in this figure is determined by the arrival time of the disturbance, but we pass from white to black four times. More precisely, we first calculate a normalized arrival time at each particle $i: \tau_{i}=t_{i} / t_{f}$, where $t_{i}$ is the pulse arrival time at grain $i$, and $t_{f}$ is the duration of the simulation. Note that $0 \leq \tau_{i} \leq 1$. Next, let $J$ be the number of times to pass from white to black. In Fig. $2, J=4$. We then calculate a gray level $g_{i}, 0 \leq g_{i}<1$ by taking the non-integer part of $J \tau_{i}$, or

$g_{i}=J \tau_{i}-$ floor $\left(J \tau_{i}\right)$.

where the floor function returns the largest integer not greater than its argument.

Fig. 2 shows that the disturbance propagation speed is strongly anisotropic when the packing is loaded the disturbance travels much more quickly in one direction that the other. The propagation velocity is determined by the first principal stress direction: it propagates quickly in the direction where normal stress is a maximum (vertically on the left of Fig. 2, horizontally on the right), and more slowly in the other direction.

\subsection{Particle interactions}

We use the simple linear spring and dashpot model for the particle-particle interaction. The normal force $F_{n}$ at each contact is

$F_{n}=-K_{n} D_{n}-\gamma_{n} \dot{D}_{n}$

where $D_{n}$ is the overlap between the two particles. In this paper, we set the normal stiffness $K_{n}=2000 p_{*}$ unless indicated otherwise. The damping coefficient $\gamma_{n}$ is chosen to obtain a restitution coefficient of about 0.92 .

The tangential force is calculated in a similar way, but with an additional condition to allow sliding. A candidate tangential force $\hat{F}_{t}$ is calculated:

$\hat{F}_{t}=-K_{t} D_{t}-\gamma_{t} \dot{D}_{t}$,

where $D_{t}$ is the integral of the tangential component of the relative motion, and $K_{t}, \gamma_{t}$ are constants analogous to $K_{n}, \gamma_{n}$. In this paper, we use $K_{t}=K_{n} / 2, \gamma_{t}=\gamma_{n} / 2$.

After calculating $\hat{F}_{t}$, we check if it satisfies

$\left|\hat{F}_{t}\right| \leq \mu F_{n}$

where $\mu$ is the friction ratio ( $\mu=0.2$ in this paper). If it does, then the tangential force is set equal to the candidate: $F_{t}=\hat{F}_{t}$. Otherwise, the contact is said to be "sliding", and $F_{t}= \pm \mu F_{n}$, choosing the sign so that $F_{t}$ and $\hat{F}_{t}$ have the same sign. If the contact slides, we set $D_{t}=-F_{t} / K_{t}$. This last step is necessary to model the sliding of the two surfaces.

This modelization is standard, but we repeat it here to draw the reader's attention to on important consequence, namely that when the contact slides, the force is incrementally non-linear, i.e., the derivative $\frac{\partial F_{t}}{\partial D_{t}}$ does not exist. To see this, let $F_{n}$ be constant, and $F_{t}=$ $\mu F_{n}>0$. If the motion is quasi-static, Eq. (4) tells us that $D_{t}=-\mu F_{n} / K_{t}<0$. Then note

$\lim _{h \rightarrow 0^{+}} \frac{F_{t}\left(D_{t}+h\right)-F_{t}\left(D_{t}\right)}{h}=-K_{n}$

since $h>0$ reduces $\hat{F}_{t}$ so that $\hat{F}_{t}<\mu F_{n}$, satisfying Eq. (5) On the other hand,

$\lim _{h \rightarrow 0^{-}} \frac{F_{t}\left(D_{t}+h\right)-F_{t}\left(D_{t}\right)}{h}=0$,

since $h<0$ increases $\hat{F}_{t}$ so that $F_{t}$ remains equal to its maximum value $\mu F_{n}$. This is the microscopic origin of the incremental nonlinearity observed in granular materials $[16]$.

In addition to the normal and tangential forces, a weak rolling resistance is applied. 
compression: $\varepsilon_{y y}=1 \%$

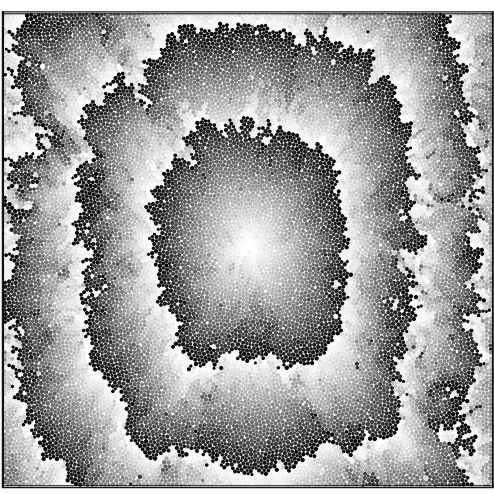

initial condition: $\varepsilon=0$

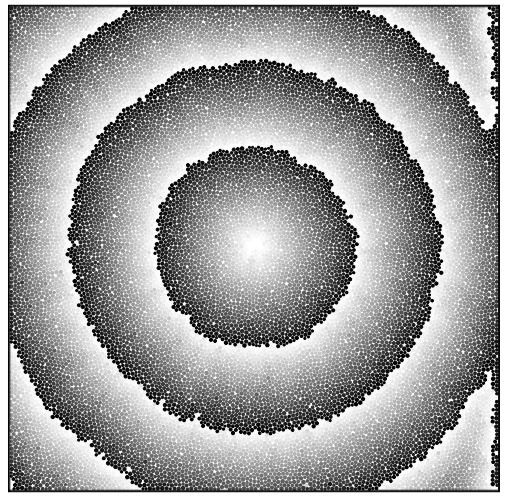

extension: $\varepsilon_{y y}=1 \%$

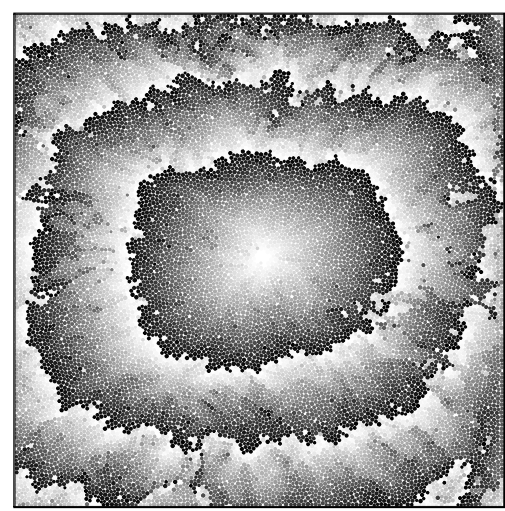

Fig. 2 Propagation of a perturbation originating in the center of the packing. The grayscale indicates when the perturbation arrival time at each particle. The strain rate is $\dot{\varepsilon}=4 \times 10^{-7} t_{a}^{-1}$ in all panels.

\subsection{Units}

The quantities $p_{*}, M_{*}$, and $\rho_{*}$ define the units used throughout this paper. In two dimensions, $\rho_{*}$ has units of mass divided by length squared, and $p_{*}$ has units of force divided by length. This implies that the unit of length is $L_{*}=\sqrt{M_{*} / \rho_{*}}$, the unit of time is $t_{*}=$ $\sqrt{M_{*} / p_{*}}$, and the unit of energy is $E_{*}=M_{*} p_{*} / \rho_{*}$.

Since we are observing acoustical phenomena, it is useful to measure time and velocity in appropriate units. A long wave propagating through a line of grains interacting according to Eq. (3) travels with a velocity

$v_{a}=d \sqrt{\frac{K_{n}}{m}}=\sqrt{\frac{4 K_{n}}{\rho_{*} \pi}}$,

where $d$ is the particle diameter and $m$ the particle mass. The first expression is general, and the second applies to disks with $m=\rho \pi d^{2} / 4$. Note that for disks (but not from spheres), this velocity is independent of particle size. The acoustic velocity enables us to define an acoustic time scale $t_{a}=L_{*} / v_{a}$, the time for a wave to travel through a line of length $L_{*}$.

An important dimensionless parameter is the ratio of the acoustic time scale to the unit of time used in the simulation:

$\frac{t_{*}}{t_{a}}=\sqrt{\frac{4 K_{n}}{\pi p_{*}}}$.

For most of the simulations in this paper, $t_{*} / t_{a} \approx 50$.

\subsection{Dimensionless Parameters}

The values of the grain stiffness, system size, and deformation rate are chosen for numerical convenience. The capabilities of the computer impose constraints on these

\begin{tabular}{lccc} 
& & Simulations & Experiments \\
\hline Container size & $L / d$ & 128 & $O(100)$ \\
Wavelength & $\lambda / d$ & $10-100$ & 20 \\
Grain stiffness & $G / p$ & 2000 & $O\left(10^{5}\right)$ \\
Strain rate & $\dot{\varepsilon} T$ & $O\left(10^{-6}\right)$ & $O\left(10^{-10}\right)$ \\
Acoustic strain rate & $\dot{\varepsilon}_{a} / \dot{\varepsilon}$ & $O\left(10^{-6}\right)-1$ & $O\left(10^{4}\right)$
\end{tabular}

Table 1 Principal dimensionless parameters characterizing the simulations presented here and the experiments[1; 3]. Explanation and method of calculation is given in the text.

parameters - for example, if there are too many particles, the simulations will take too much time. In the experiments, these parameters are subjected to other constraints. For example, the elastic properties of the beads is imposed by the material used to make them.

When interpreting the simulations, it is necessary to take these differences into account. This can be done by comparing various dimensionless parameters that characterize both the experiments and the simulations. To make our comparison concrete, we will consider two relatively small acoustic transmission experiments $[1 ; 3]$ where the experimental parameters are clearly documented.. The principal dimensionless parameters and their values are shown in Tab. 1 . As one can see certain parameters are quite close while others are different. The first two parameters describing the system size are roughly similar, but those describing the grain stiffness and the strain rate are very different. Accordingly, the effect of these last two parameters will be carefully examined in the paper.

In the rest of this subsection, we discuss the estimation of each parameter.

\subsubsection{Container size}

The experiments are done with a grain diameter $d$ of a bit less than a millimeter $(0.6 \mathrm{~mm}<d<0.8 \mathrm{~mm})$, 
in containers sizes $L$ of several centimeters $(20 \mathrm{~mm}<$ $L<60 \mathrm{~mm}$ ). This leads to a non-dimensional container size of $L / d \leq 10^{2}$ close to the value used in the simulations. This is possible because the simulations are two-dimensional while the experiments are three-dimensional. Therefore, the simulation concerns about $(L / d)^{2} \approx 10^{4}$ grains while the experiments involve $(L / d)^{3} \approx 10^{6}$.

\subsubsection{Wavelength}

The wavelength given in the papers are $\lambda \approx 10 \mathrm{~mm}[1]$ and $\lambda \approx 15 \mathrm{~mm}$ [3]. The wave length is thus roughly one order of magnitude larger than he particle diameter $(\lambda / d \approx 20)$, and a bit smaller than the container $(L / \lambda \approx 3)$. Waves of this length are easily generated in simulations.

Note that the wavelength is much larger than the grain diameter. This means that we do not acoustically probe the vibrational modes of the grains.

\subsubsection{Grain stiffness}

The confining pressures $p_{0}$ cited in these articles are $206 \mathrm{kPa}$ and $85 \mathrm{kPa}-340 \mathrm{kPa}$, while the shear modulus $G$ of glass (the material of the grains) is given as $25 \mathrm{GPa}$. This leads to a non-dimensional grain stiffness of order $G / p_{0} \sim 10^{5}$. The equivalent parameter in the simulations is $K_{n} / p_{*}=2000$, or about two orders of magnitude lower. Raising $K_{n}$ increases simulation time, because the time step used to integrate the equations of motion must be decreased. Nevertheless, we will carry out a few simulations with a higher value of $K_{n}$ to assess its effect on the results.

The choice $K_{n} / p_{*}=2000$ was motivated by the observation [17] that quasi-static flows are independent of $K_{n}$ if $K_{n}$ is several thousand times larger as the confining pressure. This threshold occurs because variations in an applied isotropic stress no longer modify the number of contacts.

\subsubsection{Strain rate}

In the experiments done with the shear cell [1], the imposed shear velocity was $0.6 \mu \mathrm{m} / \mathrm{s}$. Given the relevant container dimension $(30 \mathrm{~mm})$, this corresponds to a shear rate of $\dot{\varepsilon} \approx 2 \times 10^{-5} \mathrm{~s}^{-1}$.

Since we want to study the relation between deformation and sound, one dimensionless number is the imposed deformation per wave period $T$. The wave frequencies used with the shear were $40 \mathrm{kHz}$, leading to a period of $T=2.5 \times 10^{-5} \mathrm{~s}$. The total externally imposed shear during one wave period is thus $\dot{\varepsilon} T \approx 5 \times 10^{-10}$
On the other hand, the shear rate in the simulations is $\dot{\varepsilon}=2 \times 10^{-5} t_{*}^{-1}$, and wave periods are of order $t_{a} \approx t_{*} / 50$, leading $\dot{\varepsilon} T \approx 4 \times 10^{-7}$, or three orders of magnitude smaller. The deformation rate in the simulation is thus very large. The effect of this parameter will also be carefully examined. It is very difficult to attain such values in numerical simulations.

\subsubsection{Acoustic strain rate}

Another non-dimensional number is the ratio of the acoustical strain rate $\dot{\varepsilon}_{a}$ to the imposed strain rate. In another experiment[3], the range of displacement amplitudes of generated longitudinal waves is given as $2 \mathrm{~nm} \leq U \leq 50 \mathrm{~nm}$, with a wavelength of $15 \mathrm{~mm}$, and a frequency of $50 \mathrm{kHz}$. Estimating $\dot{\varepsilon}_{a} \approx U / \lambda T$ leads to $\dot{\varepsilon}_{a} / \dot{\varepsilon} \sim O\left(10^{4}\right) \gg 1$. In simulations, easily accessible values are in the range $O\left(10^{-6}\right) \leq \dot{\varepsilon}_{a} / \dot{\varepsilon}<O(1)$. The difference between the simulations and the experiments is very large. In the experiments, the strain is a small perturbation of the wave, whereas in the simulations, the wave is a small perturbation of the strain.

\subsection{Absorbing walls}

The wall-grain interaction is usually assumed to be the same as the grain-grain interaction, leading to a boundary that is nearly a perfect reflector of acoustic energy. When a signal crosses the packing and arrives at the opposite wall, it is reflected back into the packing, and the situation becomes much more complicated and difficult to analyze. In addition, energy generated inside the packing remains trapped inside the simulation, instead of radiating into the surroundings, as it would in an experiment. These problems become more acute as the size of the simulation in grain diameters becomes large.

A nearly absorbing wall can be implemented by decreasing the stiffness of the grain-wall interaction, and then choosing a dissipation rate so that the dominant oscillation frequency is critically damped. Specifically, the wall-grain interaction has a stiffness of $K_{n} / \sqrt{N}$ and a large damping coefficient. This choice maximizes the damping of the longest vibration mode. Incoming higher frequency waves are overdamped, and are mostly absorbed at the walls. [18]

Soft walls, however, have the disadvantage of complicating the calculation of strain during the biaxial test. The strain is usually calculated from the displacement of the external walls. But in our case, the walls are so soft that a large part of the deformation occurs between the wall and the first layer of grains. Thus we . return to the definition of the strain as the gradient of 


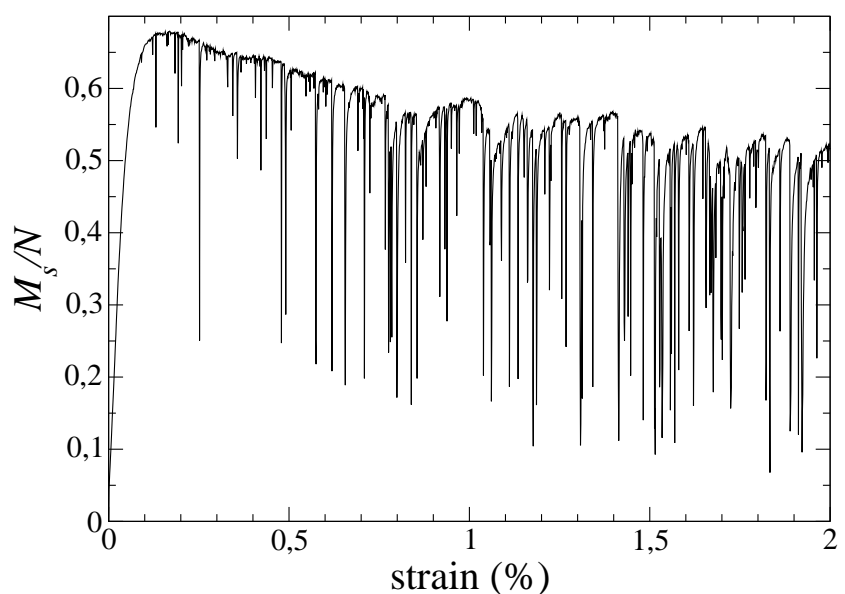

Fig. 3 Number $M_{s}$ of sliding contacts for the compression experiment shown in Fig. 1.

the displacement. The following procedure is used: The position $\left(x_{i}, y_{i}\right)$ of each grain $i$ in a reference state (initially the zero strain state) is recorded. Then, as the simulation advances, the displacement $\left(u_{i}, v_{i}\right)$ of each grain from its reference position is calculated. To obtain the $y y$-component of the strain tensor, we do a linear regression on the $y$-components, i.e., we look for $\varepsilon_{y y}, b$ such that minimizes $\sum_{i}\left(v_{i}-\varepsilon_{y y} y_{i}-b\right)^{2}$, the fit parameter $\varepsilon_{y y}$ being the strain. When $\varepsilon_{y y}$ increases above $10^{-5}$, the state of the simulation is recorded, and it defines a new reference state.

Another disadvantage of the soft walls is that acoustical signals generated by the walls are not transmitted to the packing. Therefore, we do not soften the right, mobile wall, so that we can use it to generate acoustical signals.

\section{The effect of sound on sliding}

In this section, we show that sliding contacts are very sensitive to sound waves, showing that loaded granular packings must be carefully handled (numerically and experimentally) if the effect of sliding contacts is to be studied in a meaningful way.

\subsection{Spontaneously generated noise}

Vibrations in loaded granular materials suppress sliding contacts. In Fig. 3, we show the number of sliding contacts in the compression test. The envelope of the curve resembles the stress-strain relation in Fig. 1, but the curve is punctuated by numerous sudden drops in the number of sliding contacts, followed by a rapid recovery to the original level.

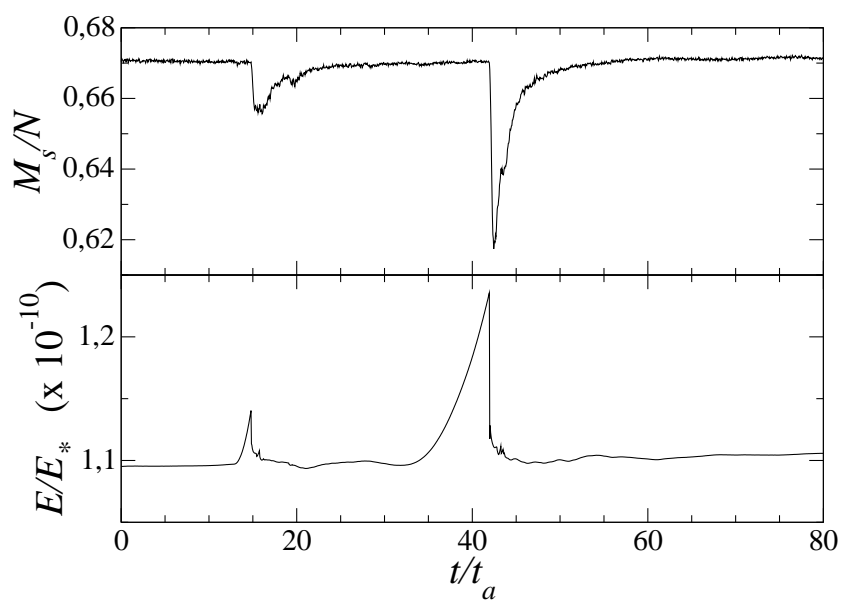

Fig. 4 Two spontaneously generated events within the biaxial test of Figs. 1 and 3. Top panel: number $M_{s}$ of sliding contacts, bottom panel: total kinetic energy $E$.

In Fig. 4, we show two such events from the same simulation as in Fig. 3, but on a magnified time scale. The top panel shows the number of sliding contacts, and the bottom panel the kinetic energy. The events shown in this figure are small compared to those in Fig. 3. Examining the larger of the two events shows a distinct sequence of events. First, the kinetic energy rises at an accelerating rate, due to an instability within the packing. Then suddenly drops, and at the same time the number $M_{s}$ of sliding contacts also drops due to a sound wave that radiates from a particular location in the sample [19]. Finally, $M_{s}$ returns slowly back to its original value.

Are these events quasi-static (governed by the global strain) or dynamic (governed by equilibrated intergranular forces and their resulting accelerations)? To examine this question, the simulation of Fig. 4 was redone with the strain rate divided by ten. The two events were again obtained, with the same energy, both beginning at precisely the same values of the global strain, indicating that such events are triggered by events generated by the quasi-static evolution of the system, as found in earlier work [19].

But the kinetic energy rise time, and the recovery time of $M_{s}$ are nearly independent of strain rate are independent of strain rate, suggesting that these are dynamic processes. This is confirmed by simulations with very stiff particles $\left(K_{n}=2 \times 10^{5}\right)$. Measured in units of $t_{a}$ (which takes into account grain stiffness), the recovery time of $M_{s}$ does not change. The energy rise times (again measured in $t_{a}$ ) are perhaps two times longer, but this difference is small compared to the factor of 10 in $t_{a}$. 


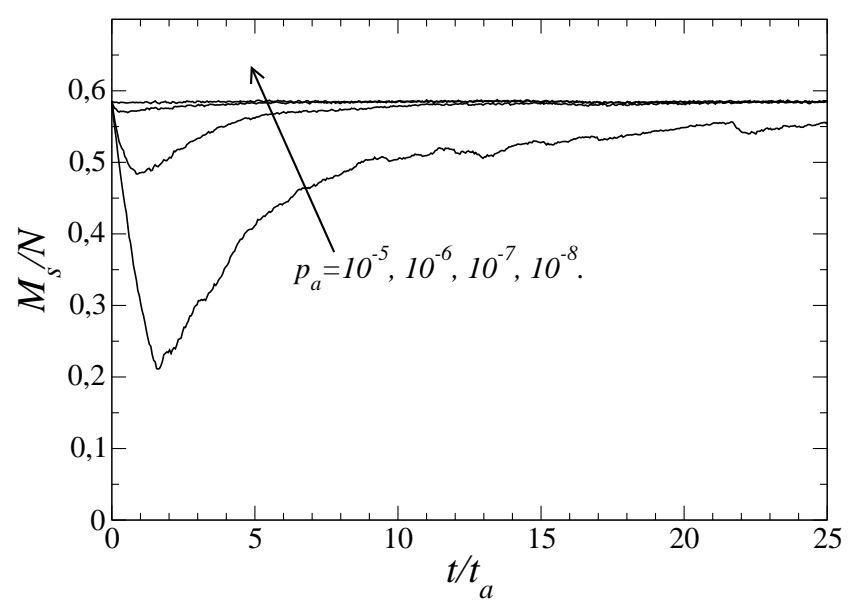

Fig. 5 Number of sliding contacts after applied pulses of varying dimensionless amplitude $p_{a}$ - see Eq. (10).

\subsection{Applied pulses}

Now we want to study this phenomena in a more controlled way. To do so, we use the technique of replaying the simulation described above. We choose a time when there are no events, and generate a pulse at the beginning of each "replay" by exerting a $\delta$-function stress on the mobile wall of varying amplitude:

$p(t)=p_{*}\left[1+p_{a} \delta(t)\right]$.

In Fig. 5, we show the effect of the pulse on the number of sliding contacts. As the pulse propagates through the material, it suppresses the sliding contacts as it goes along. This accounts for the rapid, linear drop at the beginning of the simulation. Then, there follows a slow recovery.

Note that the initial loss depends strongly on the amplitude of the pulse. At $p_{a}=10^{-5}, 2 / 3$ of the sliding contacts are lost, but the $p_{a}=10^{-8}$ pulse leaves them almost unchanged.

\subsection{A random walk model}

In this section, we present a simple model that explains why shocks cause a drop (and not an increase) in sliding contacts, followed by a slow recovery. This model is a biased random walk with a threshold. Let us suppose that a crowd of drunkards emerges at time $t=0$ from a bar at $x=0$. They then execute independent biased random walks, with a steps $\Delta x$ drawn uniformly from the interval $[\bar{\mu}-s, \bar{\mu}+s]$ with $\bar{\mu}>0$. The parameter $\bar{\mu}$ gives the drunks a mean positive velocity (they have a vague idea that they should go toward positive $x$ ), while $s$ characterizes their fluctuations about the mean drift ( $s$ being perhaps proportional to the number of drinks).

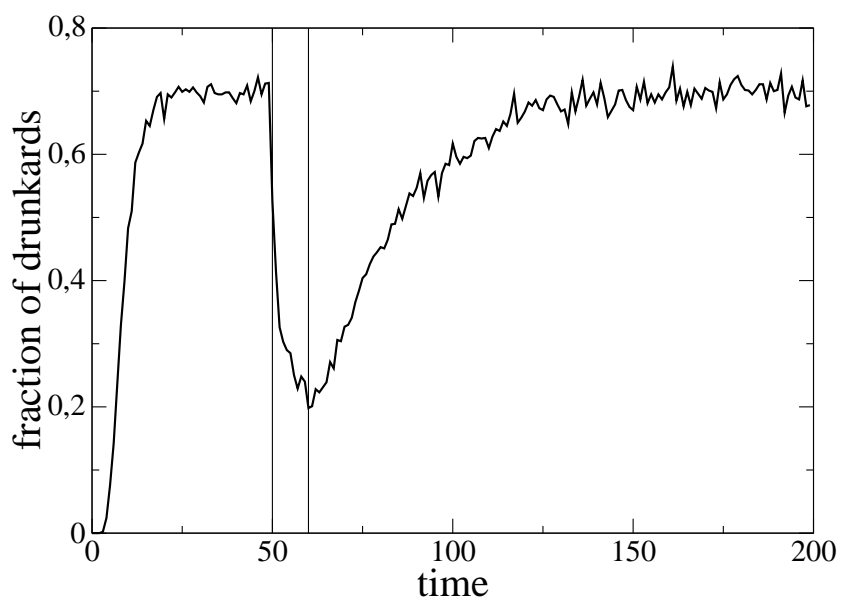

Fig. 6 Results from a biased random walk with a threshold used to model the effect of vibrations on sliding contacts. 1000 drunkards were released at $x=0$ at $t=0$. The graph shows the fraction of drunkards stuck on the fence at $x=1$. The step standard deviation is temporarily multiplied by 10 in the interval $50 \leq t<60$ (vertical lines).

There is a fence at $x=1$ that prevents the drunkards from entering the region $x>1$. When a drunkard arrives at the fence, he simple stops, and waits until he draws a negative step. He then leaves the fence.

In this model, the position of the drunkard corresponds to $F_{n}-\mu\left|F_{t}\right|$ that measures the distance of a contact from its sliding threshold. The drunkards at the fence correspond to sliding contacts. The contact forces and the drunkards act in exactly the same way: when they arrive at a barrier (the fence, or the Coulomb condition), the simply stop there until they decide to move in the other direction. The bias of the random walk represents the steady, constant imposed strain rate. This strain rate causes a steady relative motion at each contact that causes the contact forces to evolve in a constant, steady way. Finally, the random part of the drunkard's walk correspond to vibrations that propagate through the packing.

In Fig. 6 we show the fraction of drunkards at the fence during an experiment designed to mimic the passage of a wave through a loaded granular material. For $t \in[0,49]$, we set $\bar{\mu}=0.1$ and $s=0.2$. The drunkards thus choose a step distance in the interval $[-0.1,0.3]$. When they arrive at the fence, their probability of choosing a positive number, and thus remaining at the fence, is 0.75 . Note that this is very close to the maximum fraction of drunkards that are stuck on the fence.

To model the arrival of a pulse, $s$ is increased by an order of magnitude, modeling the increased fluctuations. In Fig. 6, the pulse lasts for ten steps, $50 \leq t<$ 60 . Finally, at $t=60$ we set $s$ back to its original value. The drunkards who have been scattered by the strong 


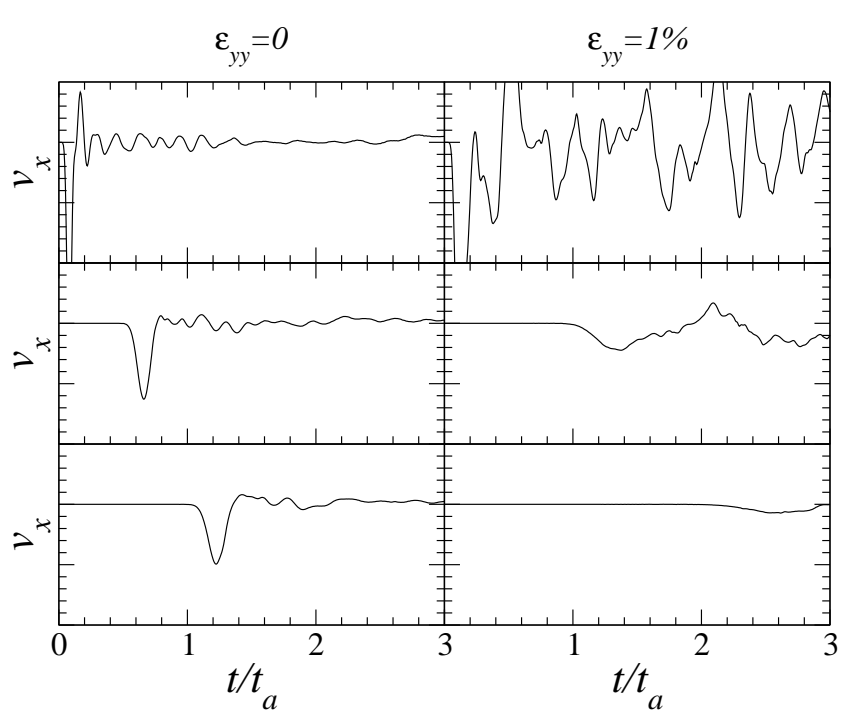

Fig. 7 The $x$-component of the perturbed velocity as a function of time during pulse transmission experiments for selected grains, with $p_{a}=10^{-10}$. Top row: a grain near right hand wall. Middle row: a grain near the center of the packing. Bottom row: a grain near the left wall. The vertical and horizontal scales on all graphs are the same. The pulse appears as a negative velocity because the initial motion of the right wall is inwards.

fluctuations, slowly return to the fence. This part of the curve models the recovery of $M_{s}$ after the passage of a pulse.

Note that the only parameter in the model is the ratio $s / \bar{\mu}$ that gives the strength of the vibration relative to the steady bias. This suggests that the lowering the strain rate (lowering $\bar{\mu}$ ) will make the sliding contacts sensitive to lower amplitude pulses. This is indeed the case. Reducing the strain rate also increases the the loss of sliding contacts. Indeed, reducing the strain by a factor of 1000 and generating a pulse with $p_{a}=10^{-8}$ suppresses more sliding contacts than the $p_{a}=10^{-5}$ pulse in Fig. 5 .

\section{Effect of sliding contacts on sound}

\subsection{Procedure}

In this section, we consider "tapping" the mobile wall with a well defined amplitude, and study how the speed of the resulting pulse. We use the pulse generation technique described above in Sec. 3.2 and in Eq. (10).

Note that the pulse amplitude $p_{a}$ can be positive or negative. If $p_{a}<0$, pressure is reduced momentarily on the right hand wall, and a rarefaction wave is generated. On the other hand, if $p_{a}>0$, a compression wave is generated.

To follow the progression of the pulse, we record the velocities of all the particles every $\Delta t=0.005 t_{a}$.
We then subtract the velocities of the $p_{a}=0$ simulation from each of the $p_{a} \neq 0$ simulations to obtain the perturbed velocity $\mathbf{v}$ of each grain. If the pulse is superimposed in a linear way on the ongoing deformation, this procedure should separate the effects of the pulse from those of the imposed deformation. Examples of the velocities obtained in this way are shown in Fig. 7. This figure shows that the pulse broadens and diminishes in amplitude as it travels, in accord with previous work[13]. When the packing is loaded (right column in Fig. 7), the dissipation and broadening become stronger, and the coda (the vibrations after the passage of the main pulse) have a much greater amplitude.

The next step is to identify the time the pulse arrives at each grain. We do this simply by locating the time where $v_{x}$, the $x$-component of the perturbed grain velocity, attains its maximum absolute value. Results for each grain, are shown for three different situations in Fig. 8. The pulse arrival times are encoded in the same way as in Fig. 2.

Fig. 8 shows the dramatic effect of the loading. The left panel shows the pulse propagation through the initial condition: the pulse propagates in an organized way. Each grain feels the arrival of the pulse at about the same time as its neighbors. When the sample is deformed (middle panel), however, the pulse is spatially fragmented - small irregular regions appear. Moreover, the size of these regions increases from right to left. This is probably due to the broadening of the pulse. If the deformation is stopped (using the procedure that will be described in Sec. 4.3) before the pulse is sent, we obtain the right panel. The pulse is no longer as fragmented as in the middle panel, but the wave fronts remain ragged.

\subsection{Pulse velocity}

The pulse arrival time of each grain is plotted as a function of the $x$-coordinate of position in the upper panels of Fig. 9. Before the load is applied $\left(\varepsilon_{y y}=0\right)$, all the points are concentrated in a single narrow band whose slope gives the pulse velocity. The loading changes two things: First, the slope of the band changes, indicating a reduced pulse velocity, and second, many grains have their maximum velocity after the passage of the pulse, showing that the "coda" is much stronger.

The points in the upper panels of Fig. 9 are confined above a diagonal line whose slope is the inverse of the pulse velocity. To calculate the pulse speed, therefore, we first divide the domain into 128 vertical strips. In each strip, we sort the particles by pulse arrival time (time of maximum $v_{x}$ ). Then we identify the grain with 

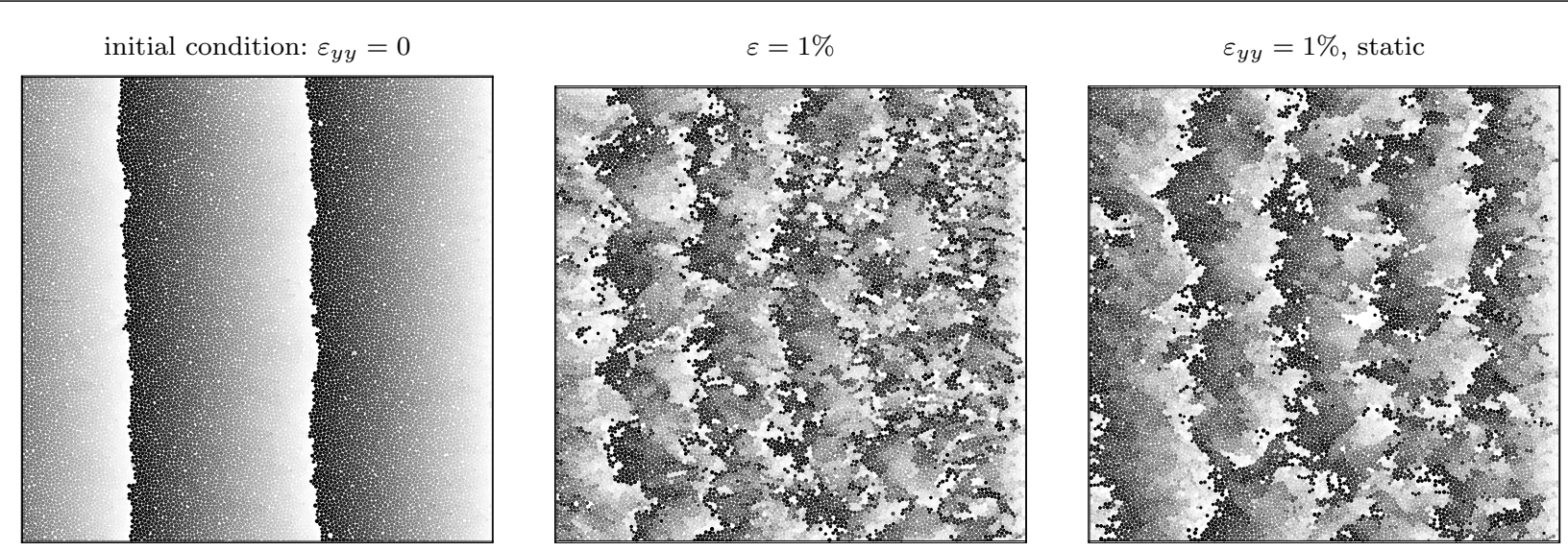

Fig. 8 Time of pulse arrival at each grain. The pulse arrives at light particles before dark ones, but we pass from white to black six times during the simulation. The simulation time is $3 t_{a}$, so the time between two successive wavefronts is $t_{a} / 2$.

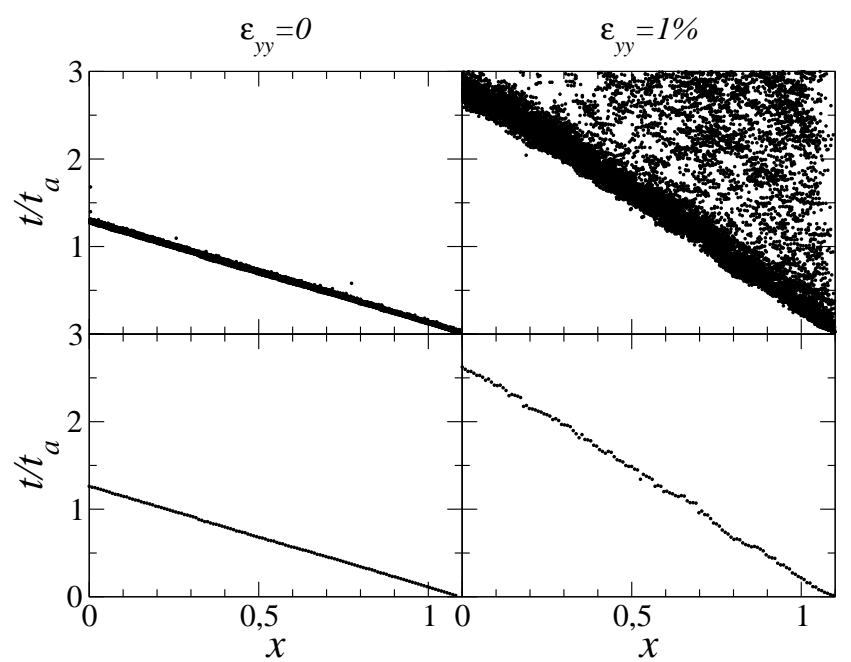

Fig. 9 Two examples of the measurement of pulse velocity. Upper panels: the time of the velocity maximum for each particle. Lower panels: Third smallest pulse arrival time for the grains in thin vertical strips, used to calculate pulse velocity. These data come from the left and center panels of Fig. 8.

the third smallest arrival time in each strip. The results are shown in the lower panels of Fig. 9. We fit the data of these lower panels to obtain a pulse speed.

\subsection{The static limit}

As discussed in Sec. 2.6, the zero strain rate limit is relevant to experiments. Unfortunately, one can not abruptly slow or stop the applied strain for the sliding contacts are extremely sensitive to vibrations, and one would like to preserve them in order to study their effect. We therefore approach the static limit in two steps.

The first step is to reload a checkpoint, and "replay" it at a reduced strain rate, multiplying all velocities by a factor $\gamma<1$ before beginning the simulation. Since the initially simulation is quasi-static, this procedure should generate a new equilibrium state with a strain rate multiplied by $\gamma$. The resulting state is not precisely in equilibrium for some forces, especially the forces involving the soft walls, do depend on velocity. Therefore an adjustment occurs that suppresses sliding contacts. These sliding contacts can be recovered by applying an addition strain of $\Delta \varepsilon=2 \times 10^{-6}$. In this way, we obtain states for $\gamma=0.1,0.01,0.001$.

The second step is to reload the $\gamma=0.001$ state, and then stop the walls as gently as possible. Specifically, the velocity of the upper wall is slowly decreased to zero:

$v_{\text {wall }}=v_{0}\left(1-\cos \pi t / t_{s}\right)$

where $t_{s}$ can be given various values. Finally, the packing is allowed to radiate its remaining energy over a time of about $100 t_{a}$.

In Fig. 10, we show the pulse speed in samples obtained in the way described above. First of all, let us examine the effect of reducing the strain rate by a factor of $\gamma$. These simulations change most significantly for $p_{a}>0$. We observe a rapid jump in velocity at a value of $p_{a}$ that appears to be proportional to $\gamma$; at $p_{a} \approx \gamma / 10^{-5}$. We interpret this drop as corresponding to the amplitude where the pulse becomes strong enough to suppress the sliding contacts as it travels. As the strain rate drops, weaker pulses can suppress the sliding contacts. This interpretation is confirmed by examining the number of sliding contacts.

Note that these simulations yield a pulse speed independent of the sign of $p_{a}$ for very small $p_{a}\left(\left|p_{a}\right|<10^{-9}\right)$. This indicates that the pulses are linear. They are too weak to suppress any sliding contacts, that are maintained by the imposed shear rate. 


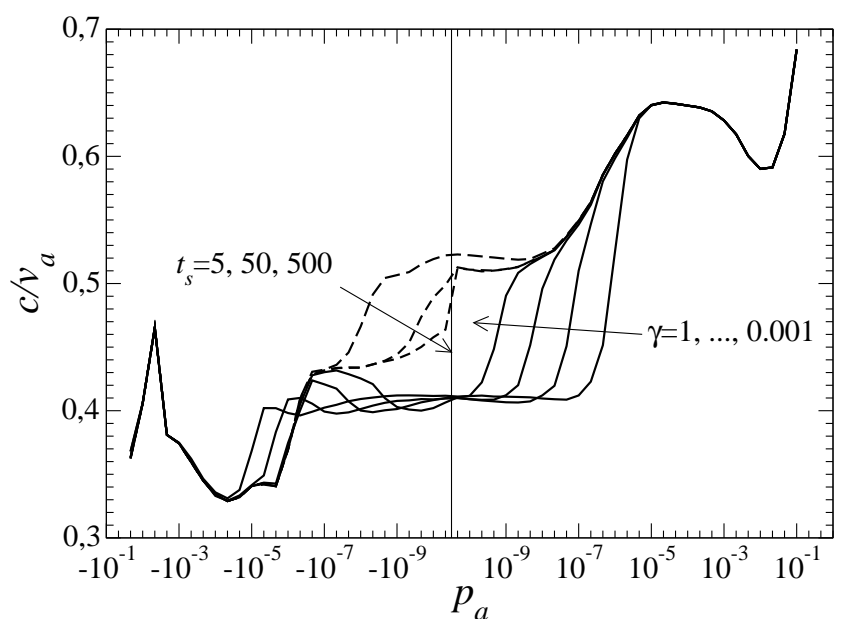

Fig. 10 Convergence toward the static limit. The curves marked $\gamma=1 \ldots 0.001$ show the result of reducing the strain rate by a factor $\gamma$. The curves marked $t_{s} / t_{a}=5 \ldots 500$ show the effect of stopping the strain rate over a duration $t_{s}$. All states are prepared from the $\varepsilon_{y y}=1 \%$ checkpoint of the compression test.

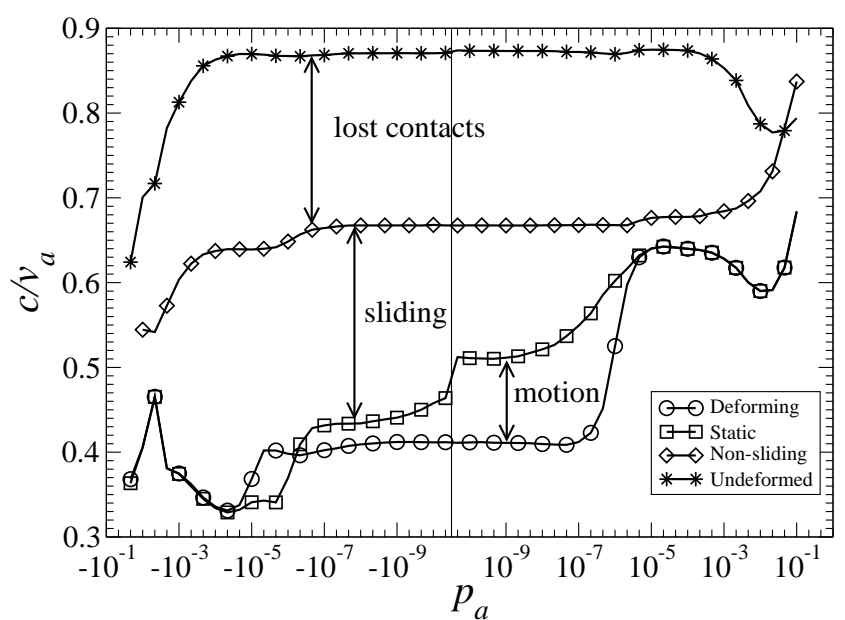

Fig. 11 Physical mechanisms affecting pulse speed. The two lower curves are from Fig. 10.

Now let us examine the second series of samples, obtained by stopping the $\gamma=0.001$ sample over a time $t_{s}$. In these samples, the strain rate vanishes, so that any pulse, no matter how weak, can suppress sliding contacts. As a result, the pulses are more rapid than those with finite strain rate. But the most interesting feature is that the pulse speed depends on the the sign of $p_{a}$, even at very small pulse velocities. Furthermore, it seems that if we could stop the strain rate with an infinite gentleness, the pulse speed would be discontinuous at $p_{a}=0$. This is an acoustical manifestation of the incremental non-linearity of sliding contacts.

\begin{tabular}{lcccc}
\multirow{2}{*}{ Physical process } & \multicolumn{2}{c}{ Compression } & \multicolumn{2}{c}{ Extension } \\
& $p_{a}<0$ & $p_{a}>0$ & $p_{a}<0$ & $p_{a}>0$ \\
\hline Loss of contacts & -0.233 & -0.236 & -0.038 & -0.037 \\
Sliding contacts & -0.260 & -0.179 & -0.090 & -0.107 \\
Non-zero strain rate & -0.034 & -0.115 & -0.032 & -0.014 \\
Total & -0.527 & -0.530 & -0.160 & -0.158
\end{tabular}

Table 2 Relative loss of sound speed $\Delta c / c_{0}$, due to the three physical processes tested in the simulations. The sound speeds are measured at $\left|p_{a}\right|=10^{-9}$.

\subsection{Physical processes affecting pulse speed}

Fig. 11 separates the different physical processes affecting the pulse speed. Four different types of experiments are shown:

1. Deforming. The pulse propagates while the deformation is ongoing (circles in Fig. 11).

2. Static. The strain rate is slowly brought to zero as described in Sec. 4.3 before generating the pulse (squares).

3. Non-sliding. The same as the static experiment, except that we set the friction ratio to 2 (instead of 0.2 ) to suppress the effect of sliding contacts (diamonds).

4. Undeformed. The pulse travels through the packing before any deformation is applied (stars).

The difference between the various experiments enables us to isolate the various physical effects that reduce the pulse speed. For example, the only difference between the deforming and static experiments is that imposed strain rate is nonzero in the first case, but zero in the second case. Thus the difference between the two reveals the effect of the motion.

The difference between the static and non-sliding experiments is that the first contains sliding contacts, or contacts close to the sliding threshold, whereas the second case does not. Thus the difference between these two shows the effect of sliding contacts.

Finally, neither the non-sliding experiment nor the undeformed experiments contain sliding contacts, but their contact networks do differ. The application of the load creates an anisotropic contact distribution, whereas the undeformed state has a nearly isotropic distribution. Thus the effect of this change is given by the difference between these two cases.

The contribution of all these effects is summarized in Tab. 2. The loss in sound speed at each step is given as a fraction of the sound speed $c_{0}$ in the undeformed sample. Also appearing in Tab. 2 are the results of the same experiments done on the extension experiments. In this case, the changes in sound speed are much weaker. 


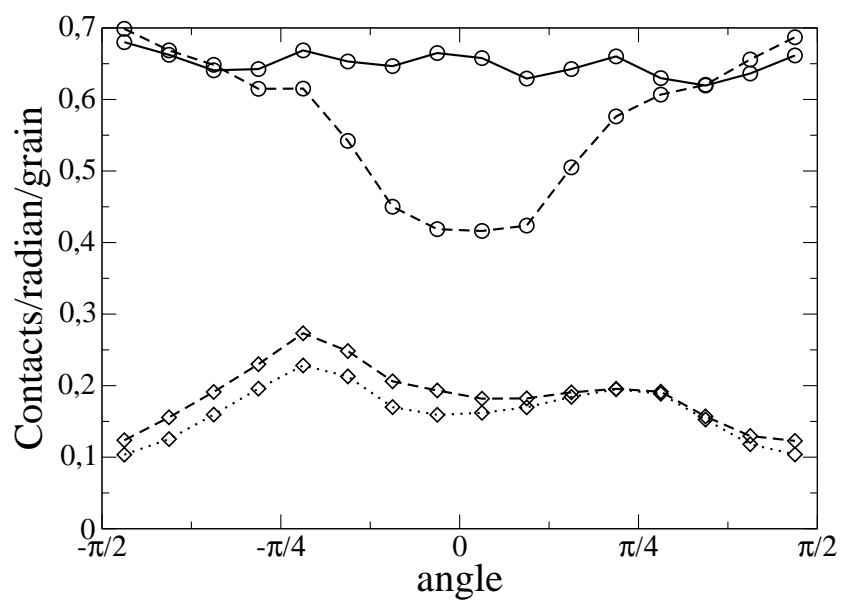

Fig. 12 Orientation of contacts: Horizontally oriented contacts: $\theta=0$, vertically oriented contacts: $\theta= \pm \pi / 2$. Circles: all contacts, diamonds: sliding contacts. Solid line: undeformed sample, Dashed line: deformed sample $\left(\varepsilon_{y y}=1 \%\right)$ with non-zero strain rate. Dotted line: loaded sample with zero strain rate. Contacts with the walls are excluded.

\subsection{Contact network anisotropy}

To confirm the interpretations given in Fig. 11 and Tab. 2, angular distribution of the contacts. The changes and anisotropy of the pulse speed should correspond to changes in the contact network.

In Fig. 12, we show the number of contacts at a given angle, per radian and per grain. The contact angle $\theta$ is the angle between the $x$-axis and the line of centers. The two states used to construct Fig. 11 are used, and both total and sliding contacts are considered.

Let us consider first the total contacts. The global stiffness of the packing should be proportional to the number of contacts, and the sound speed is proportional to the square root of the stiffness. Fig. 12 shows that the loading depletes horizontal contacts but not vertical ones: at $\varepsilon_{y y}=1 \%$, about a third of the horizontal contacts have been lost, but none of the vertical ones. Assuming that the pulse is carried mainly by the contacts aligned in the direction of propagation, we find that the loss of contacts should reduce the sound speed by a factor of about $1-\sqrt{2 / 3} \approx 0.184$ in the compression test, but not at all in the extension test. The observed reductions are a bit larger, the difference between the two could be explained by appealing to the non-affine motion of the grains that reduce the stiffness, and are probably more important when the coordination number is smaller [].

Now let us consider the sliding contacts. Fig. 12 shows that the largest number of sliding contacts have $\theta= \pm \pi / 4$. There is some asymmetry: there are more sliding contacts for $\theta<0$ than for $\theta>0$. Bringing the strain rate to zero reduces this asymmetry.
If we consider the fraction of contacts at a given $\theta$ that are sliding, a different picture emerges. Due to the depletion of horizontal contacts, nearly half the contacts are sliding at $\theta=0$, between a half and a third at $\theta= \pm \pi / 4$, and only about one seventh at $\theta= \pm \pi / 2$. Turning to now to Tab. 2, we see that the sound velocities have the same isotropy. The reduction in sound speed is between two and three times greater in the compression test than in the extension test.

Note that there is no theoretical result for the influence of sliding contacts. Neither are they mentioned when explaining experimental results. These results suggest that they should be taken into account.

The anisotropy of the wave propagation speed can also be understood by considering the loaded granular material as a superposition of a strong contact network and a weak one [20]. The strong contact network consists of contacts with above average normal force and acts as an anisotropic solid, with the majority of the contacts aligned along the direction of the first principal stress. The weak contact network consists of contacts with below average normal force, and is nearly isotropic and dissipative. In the extension experiments, the pulses travel along the direction of the first principal stress and are carried by the strong network. Sliding contacts have a minimal effect because most of them are in the weak network. In the compression experiments, however, the pulses are carried by the weak network, and sliding contacts have a major effect.

\subsection{Effect of particle stiffness}

In Fig. 13, we show the effect of particle stiffness. Rescaling the pulse velocities with $v_{a} \propto \sqrt{K_{n}}$. is sufficient to bring the curves close together. The discontinuity of the speed when $p_{a}$ changes sign is visible in both cases, indicating that the use of relatively soft $\left(K_{n}=2000 p_{*}\right)$ grains captures the essential physics.

The harder particles are technically more difficult to handle. The weak pulses do not propagate through the material, and so the velocities are estimated using only the right half of the simulation.

\subsection{Dependence on load}

Do the changes in Fig. 11 appear only near failure or are they also visible in lightly loaded situations? To answer this question, we show in Fig. 14 how the pulse speed changes as the load is increased. As one can see, the decrease in sound velocity occurs in a continuous way, rapidly at first, and then more slowly. The most 


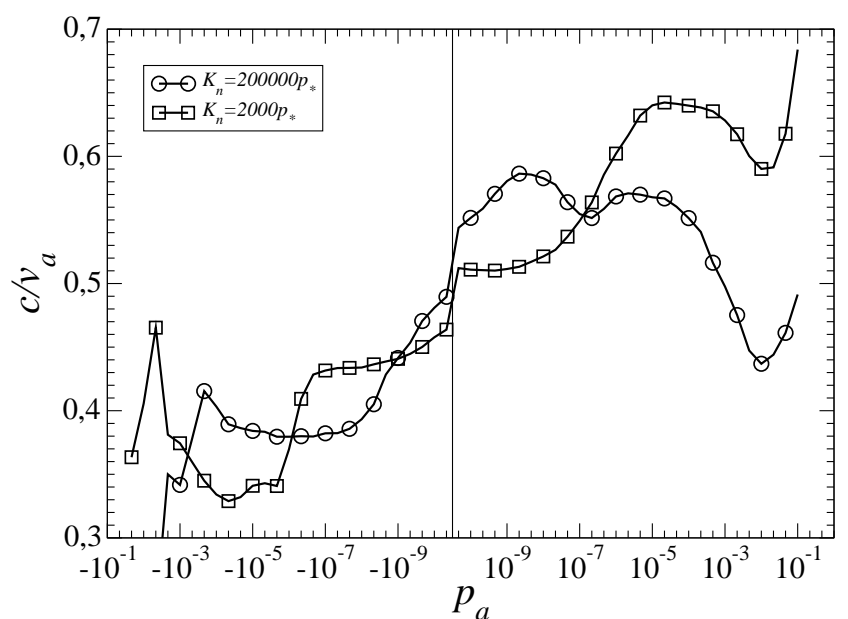

Fig. 13 Effect of particle stiffness on pulse velocity. Both curves are obtained in the same way: After imposing a deformation $\varepsilon_{y y}=1 \%$, the samples are then brought to a stop. The curve $K_{n}=2000 p_{*}$ is obtained from a appears in Figs. 10 (curve $\left.t_{s}=500\right)$ and 11 (curve Static). Note that $K_{n}$ appears in the definition of the acoustic velocity $v_{a}$.

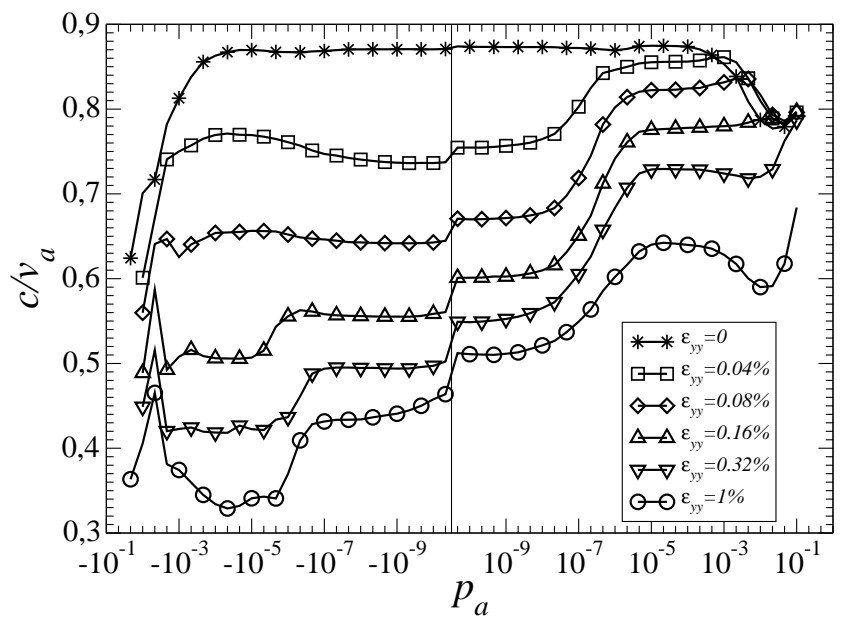

Fig. 14 Pulse speeds as a function of imposed deformation. All simulations are "static", that is the strain rate is gently brought to zero, as described above.

important point of this figure, however, is that the discontinuity at $p_{a}=0$ appears as soon as the loading begins. It is not a consequence of being close to the failure threshold. One can furthermore see that sound speed is not related in a simple way to the number of sliding contacts. The sound speed decreases monotonically between $\varepsilon_{y y}=0$ and $\varepsilon_{y y}=1 \%$, but the number of sliding contacts is strongly non-monotonic (see Fig. 3).

\section{Discussion and conclusion}

To conclude this paper, we examine in Sec. 5.1 some experiments for evidence of the reduction of sound speed due to sliding contacts. The experimental results are

\begin{tabular}{ccc} 
& p-wave & s-wave \\
Compression [4] & & -0.057 \\
\hline vertical & +0.054 & -0.049 \\
horizontal & $-0.134,-0.107$ & \\
Extension [4] & & -0.121 \\
\hline vertical & -0.168 & -0.007 \\
horizontal & $+0.097,+0.080$ & \\
Oedometric [5] & & +0.616 \\
\hline vertical & +0.473 & $+0.384,+0.419$
\end{tabular}

Table 3 Changes in sound speed $\Delta c / c_{0}$ measured in experiments $[4 ; 5]$. Here $=\Delta c=c_{0}-c_{f}$, where $c_{f}$ is the sound speed for the largest available shear stress. The oedometric data are for the "decompaction" initial condtion [5]. When two speeds are given, it is because there are two independent directions or polarizations.

indecisive: they neither exclude nor confirm the action of sliding contacts. An anisotropic reduction in sound speed is indeed observed, but an explanation in terms of a simple change in the contact network could be envisaged.

We then recall in Sec. 5.2 the assumption that the modelization of sliding contacts used in the simulations applies at acoustic time scales. We propose an alternative where sliding contacts would not affect the acoustics, but still govern the quasi-static stress-strain relation.

The paper concludes with Sec. 5.3 where the distinctive acoustic signature of sliding contacts is discussed. The modelization used for sliding contacts could be checked by looking for this signature in experiments.

\subsection{Is there experimental evidence of sliding contacts?}

First of all, in the study used to dimension the simulations [1], the loss of contacts could explain the reduction of the p-wave speeds, but only have of the s-wave speeds. This paper concerns only p-waves, and there are other ways of explaining the slowing of s-waves. For example, the shear modulus is much more sensitive to non-affine motions than the bulk modulus [21;22]. An increase in these motions would affect mainly the s-wave speed.

But it is the experiments of Agarwal and Ishibashi[4] that correspond most closely to the situation studied in the paper. They measured sound speed along different paths during stress-controlled compression and extension tests. We also use the more recent experiments of Khidas and Jia [5] who measured sound speeds in an oedometric test. The most relevant results are summarized in Tab. 3.

The experiments show the same anisotropy of the sound speed as the simulations: the sound speeds are 


$\begin{array}{lccc} & \text { Compression } & \text { Extension } & \text { Oedometric } \\ \text { Initial } & -0.042,-0.016 & -0.087,-0.096 & 0.018 \\ \text { Final } & 0.154,0.150 & 0.188,0.165 & 0.145\end{array}$

Table 4 Anistropy for the experiments [4; 5], as calculated using Eq. (12), for p-waves.

$\begin{array}{lcc} & p_{a}<0 & p_{a}>0 \\ \text { Initial } & 0.002 & -0.003 \\ \text { Non-sliding } & 0.228 & 0.228 \\ \text { Static } & 0.533 & 0.372 \\ \text { Deforming } & 0.562 & 0.563\end{array}$

Table 5 Anisotropy from the simulations, for different numerical experiments. The names of the the numerical experiments are taken from Fig. 11, and the data from Tab. 2.

larger in the direction of the principal stress (vertical in the compression and oedometric test, horizontal in the extension test).

The experimental results of Tab. 3 can be compared to the numerical results of Tab. 2. In general, we see that the changes of velocity are greater in the simulations than in the experiments, suggesting that sliding contacts do not need to be invoked to explain the observations. Indeed, the change in fabric ("Loss of contacts" in Tab. 2) is more than sufficient to account for the observed change in sound speed.

A more detailed comparison is made difficult by the augmentation of the experimental sound speeds with confining pressure, which does not exist in the simulations. To get around this difficulty, we consider the anisotropy of the sound speeds:

$A=2 \frac{c_{1}-c_{2}}{c_{1}+c_{2}}$

Here, $c_{1}$ is the sound speed along the direction of the first principal strain (vertical for the compression test, horizontal for the extension tests), and $c_{2}$ is the speed measured in the other direction. The anisotropy for different simulations and experiments is given in Tabs. 4 and 5. Again, an explanation of the anisotropy observed in experiments does not require appealing to sliding contacts We conclude therefore that these experiments do not show evidence for sliding at acoustic time scales.

\subsection{Alternative models of sliding contacts}

Sliding contacts are necessary to obtain realistic stressstrain curves in a discrete element simulation of a triaxial test. It is therefore difficult to deny that sliding contacts simply do not exist in the experiments. Perhaps they determine the stress-strain curve but not the acoustic properties.
As we have noted in Sec. 3, sliding contacts are very delicate and can be disrupted by applied vibrations. It is possible that vibrations in the experiments suppress sliding contacts, while still allowing sliding to occur.

Another possibility is that their modelization is not correct at acoustic time scales. As pointed out in the introduction, the work in this paper depends on the strong hypothesis that the solid friction law discussed in Sec. 2.4 is valid at acoustic time scales.

Let us propose an alternative formulation of the solid friction law that introduces a time scale $\tau$ related to the activation of slip. We let $F_{t}=-K_{t} D_{t}-\gamma_{t} \dot{D}_{t}$ as in Sec. 2.4, but

$\dot{D}_{t}=v_{t}-\Theta\left(\left|D_{t}\right|-\left|D_{t}^{c}\right|\right) \frac{D_{t}-D_{t}^{c}}{\tau}$.

Here $v_{t}$ is the tangential component of the relative velocity traditionally used in the tangential force law, $D_{t}^{c}=\mu F_{n} / K_{t}$ is the critical value of $D_{t}$ at which the contact begins to slide. The Heaviside function $\Theta$ assures that the second term is active only when $D_{t}$ exceeds $D_{t}^{c}$. The modelization used in the paper is equivalent $\tau \rightarrow 0$, so that contact react instantaneously to any possible violation of the condition $\left|F_{t}\right| \leq \mu F_{n}$. But if $\tau$ had a value intermediate between the imposed strain rate and the acoustic time scale, contacts would slide nearly instantaneously at long time scales, giving the correct stress-strain curve, but behave as non-sliding contacts as far as sound waves are concerned.

\subsection{The acoustic signature of sliding contacts}

The discussion in Sec. 5.1 does not show that sliding contacts have no effect; it shows that sliding contacts are not needed to explain any of the experimental results.

But what would be an clear sign of their presence? One could look for the characteristic signature of sliding contacts that appears throughout this paper: incremental non-linearity. At low or zero strain rates, the pulse speed depends on the sign of $p_{a}$, even when $p_{a}$ is very small. This effect could be searched for experimentally.

In many experiments, a wavelet that is a high order derivative of a Gaussian peak is used. Would the characteristic signature of sliding contacts still appear? To answer this question, we applied a fourth derivative of the Gaussian

$f(t)=p_{a}\left(3-6 \tau^{2}+\tau^{4}\right) e^{-\tau^{2} / 2}$.

to the right wall, and measured the progression of the pressure signal through the material. The measured pulse speeds are shown in Fig. 15. As one can see, the 


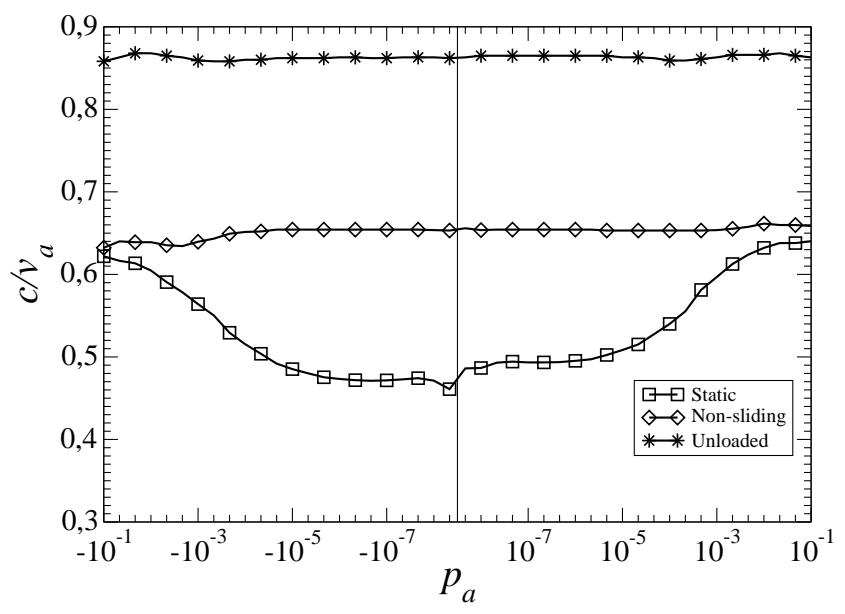

Fig. 15 Speed of wavelets in the granular material.

discontinuity of the speed is reduced but still visible. Thus we expect that this non-linearity could be detected experimentally, if contacts were sliding at acoustic time scales. In this way, the applicability of the model could be checked experimentally.

\section{Disclosures}

The author thanks Jim Jenkins for pointing out the existence of Ref. [4], and D. Imbert, Y. Le Gonidec, and L. Le Marrec for discussions.

This work was supported by the ANR grant STABINGRAM No. 2010-BLAN-0927-01. The author confirms that the work presented in this paper has never been submitted to any other journal or to any conference proceeding.

\section{References}

1. Y. Khidas and X. Jia, Probing the shear-band formation in granular media with sound waves, Phys Rev E 85051302 (2012).

2. V. Yu. Zaitsev, P. Richard, R. Delannay, V. Tournat and V.E. Gusev, Pre-avalanche structural rearrangements in the bulk of granular medium: Experimental evidence EPL $\mathbf{8 3} 64003$ (2008).

3. X. Jia, Th. Brunet, and J. Laurent Elastic weakening of a dense granular pack by acoustic fluidization: slipping, compaction, and aging Phys Rev E 84 020301R (2011).

4. T.K. Agarwal and I. Ishibashi, Anisotropic Elastic Constants of Granular Assembly from Wave Velocity Measurements, Advances in Micromechanics of Granular Materials, H.H. Shen et al (eds) p.51-60 (1992)

5. Y. Khidas, X. Jia, Anisotropic nonlinear elasticity in a spherical-bead pack: Influence of the fabric anisotropy, Phys Rev E 81021303 (2010)

6. G. Michlmayr and D. Or, Mechanisms for acoustic emissions generated during granular shearing, Granular Matter 16 627640 (2014).
7. O. Mouraille, W. A. Mulder, S. Luding, Sound wave acceleration in granular materials JSTAT P07023 (2006).

8. O. Mouraille, O. Herbst and S. Luding, Sound propagation in isotropically and uni-axially compressed cohesive, frictional granular solids Engineering Fracture Mechanics 76 786-791, (2009).

9. O. Mouraille and S. Luding, Sound wave propagation in weakly polydisperse granular materials [ Ultrasonics 48 (6-7) 498-505, 2008

10. O. Moraille, Sound Propagation in dry granular materials: discrete element simulations, theory, and experiments, University of Twente (2009).

11. Y. Sakamura and H. Komaki, Numerical simulations of shock-induced load transfer processes in granular media using the discrete element method Shock Waves 22 57-68 (2012).

12. S. Melin, Wave propagation in granular assemblies, Phys Rev E 49 2353-2361 (1994).

13. E. Somfai, J.-N. Roux, J.H. Snoeijer, M. van Hecke, and W. van Saarloos, Elastic wave propagation in confined granular systems Phys Rev E $\mathbf{7 2} 021301$ (2005).

14. J. Lopolds, G. Conrad, and X. Jia, Onset of Sliding in Amorphous Films Triggered by High-Frequency Oscillator Shear, Phys Rev Lett 110248301 (2013).

15. S. van den Wildenberg, M. van Hecke, and X. Jia, Evolution of granular packings by nonlinear acoustic waves, EPL 101 14004 (2013).

16. F. Darve and F. Nicot, On incremental non-linearity in granular media: phenomenological and multi-scale views, Int $J$ Num and Analytic Meth in Geomechanics 29 1387-1409 (2005).

17. J.N. Roux and F. Chevoir, Dimensional Analysis and Control Parameters, in Discrete-element modeling of Granular Materials, F. Radja and F. Dubois, eds. ISTE, J. Wiley p. 199-228 (2011).

18. S. McNamara, Absorbing boundary conditions for granular acoustics, in III International Conference on Particle based Methods - Fundamentals and Applications, PARTICLES 2013, M. Bischoff, E. Oñate, D.R.J Owen, and P. Wriggers, eds (2013).

19. P. Welker and S. McNamara, Precursors of failure and weakening in a biaxial test, Granular Matter 13 93-105 (2011)

20. F. Radja, D.E. Wolf, M. Jean, and J.-J. Moreau, Bimodal Character of Stress Transmission in Granular Packings, Phys Rev Lett 8061 (1998).

21. I. Agnolin and J.-N. Roux, Internal states of model isotropic granular packings. III. Elastic properties, Phys. Rev. E 76 061304 (2007).

22. H.A. Makse, N. Gland, D.L. Johnson, and L.M. Schwarz, Why Effective Medium Theory Fails in Granular Materials, Phys. Rev. E 83 5070-5073 (1999) 